\title{
Trésors des îles Salomon. La collection Conru de Kevin
} Conru et Deborah Waite

\section{Gilles Bounoure}

\section{(2) OpenEdition}

1 Journals

Édition électronique

URL : http://journals.openedition.org/jso/6124

DOI : $10.4000 /$ jso.6124

ISSN : $1760-7256$

\section{Éditeur}

Société des océanistes

\section{Édition imprimée}

Date de publication : 15 décembre 2010

Pagination : 262-264

ISBN : 978-2-85430-027-7

ISSN : 0300-953x

\section{Référence électronique}

Gilles Bounoure, "Trésors des îles Salomon. La collection Conru de Kevin Conru et Deborah Waite», Journal de la Société des Océanistes [En ligne], 130-131 | 2010, mis en ligne le 15 décembre 2010, consulté le 21 septembre 2020. URL : http://journals.openedition.org/jso/6124 ; DOI : https://doi.org/ $10.4000 / j s 0.6124$

\section{(C) Tous droits réservés}


plus ou moins de soin dans la réalisation (ainsi de la réédition en 2009 de $A$ Naturalist among the HeadHunters... de Charles Morris Woodford [1890], avec une carte illisible faute d'avoir été reproduite dépliée). En France même, L'Harmattan a inauguré une collection de « fac-similés océaniens » dirigée par Frédéric Angleviel, dont l'intérêt et la qualité ont été soulignés chaque fois que possible dans ces colonnes.

Les éditions Fines Mundi, fondées il y a un peu plus d'un lustre par le Dr Rolf Kittler et spécialisées dans l'ethnographie et les récits de voyages, ont diverses particularités méritant qu'on les signale aux océanistes. Elles ont pour origine un atelier de reliure, dirigé par la même famille depuis trois générations, et dont les activités se poursuivent notamment grâce aux rééditions de Fines Mundi. Ainsi s'explique que ces facsimilés soient tous proposés sous une, deux, trois ou quatre reliures différentes, plus ou moins luxueuses d'aspect et sans grand surcoût de l'une à l'autre, mais avec des qualités de finition et de robustesse passablement rares aujourd'hui. Les ouvrages proposés par le catalogue, qui compte déjà plus de 1600 titres, figurent eux aussi le plus souvent au nombre des raretés ou des classiques fort coûteux parce que convoités par beaucoup de bibliothèques et de chercheurs. Plusieurs dizaines d'entre ceux qui concernent l'Océanie peuvent être qualifiés d' ' introuvables » auprès des libraires et de nombreuses bibliothèques, du fait soit de leur langue (l'allemand le plus souvent, mais il y a aussi des volumes en anglais, en français, etc.), soit de leur ancienneté, soit du faible tirage de leur première édition.

Du livre de Georg Thilenius ainsi réédité, et ici complet de ses deux parties souvent disjointes, on se bornera à rappeler quelle source documentaire unique il constitue pour les « enclaves polynésiennes » situées au nord et à l'est de la Nouvelle-Guinée (Ninigo, Kaniet, Nuguria, Sikaiana notamment), ainsi que pour l'archipel de l'Amirauté, également visité par le médecin-ethnographe délégué par l'Académie des sciences de Berlin, au cours de son périple de 18981899. Évidemment intéressé au premier chef par tous les indices possibles d'influences culturelles ou de migrations de nature à conforter les théories diffusionnistes alors en vogue chez les savants allemands, et très préoccupé aussi d'anthropologie physique pour des raisons similaires, il ne s'en est pas moins livré à des investigations attentives dans des domaines très variés, navigation, horticulture, magie, mythes, linguistique etc., au moment même où ces îles entraient dans un processus de "dépopulation » (et de déculturation) dont s'alarmerait le gouvernement australien au cours des années 1920.

Plus remarquablement encore, le nom de Thilenius figure également au catalogue de Fines Mundi avec la réédition quasi intégrale des Ergebnisse der SüdseeExpedition 1908-1910, herausgegeben von Dr. G. Thilenius, etc., soit tous les titres publiés entre 1908 et 1938 à Hambourg chez Friederichsen \& De Gruyter, souvent en plusieurs tomes. Il y manque encore le volume Nova Britannia publié en 1954 à Berlin par Otto Reche, mais à ce travail d'anthropologie physique qui se ressent beaucoup des vues « raciologiques » de cet auteur (et que ne possède pas la BNF !), on doit évidemment préférer son premier grand travail, Der KaiserinAugusta-Fluss, publié en 1913, et dont la reproduction par Fines Mundi est impeccable, exception faite des vues photographiques, nettement plus précises sur la publication originale.

De Unter den Papuas de Bruno Hagen (1899, qui manque à la BNF) aux Südsee-arbeiten d'Otto Finsch (1914) en passant par Eine Forschungsreise im Bismarck-Archipel de Hans Vogel (1911) ou le rarissime album de Gunnar Landtman Ethnographical Collection from the Kiwai District of British New Guinea in the National Museum of Finland (1933, qui manque à la BNF), ce sont plusieurs dizaines de livres d'intérêt exceptionnel et souvent réédités pour la première fois que propose désormais Fines Mundi, avec une qualité de réalisation remarquable à des tarifs aussi modérés. De l'exhumation de ces trésors documentaires ainsi mis à la portée d'un plus grand nombre de bibliothèques et d'océanistes, il n'est pas absurde d'attendre un certain renouveau des recherches, dont on devra alors tenir quelque gré à Rolf Kittler et à Fines Mundi.

\section{RÉFÉRENCE CITÉE}

WoODFORD Charles Morris, 1890. A Naturalist among the Head-Hunters: Being an Account of three Visits to the Solomon Islands in the years 1886, 1887, 1888, Kessinger Publishing.

Gilles BounOure, Paris

Conru Kevin et Deborah WaIte, 2008. Trésors des îles Salomon. La collection Conru, photographies de Hugues Dubois, Milan, Éditions 5 Continents, 200 p., bibliogr., cartes, 130 illustrations.

Les publications illustrées dévolues aux arts traditionnels des îles Salomon restant fort peu nombreuses, on ne trouvera pas mauvais qu'un collectionneur et marchand ait donné l'occasion à l'une des meilleures spécialistes actuelles de ces arts de décrire la centaine de pièces qu'il avait rassemblées au moment de la préparation de ce livre. Elles offrent un échantillon assez éloquent de la diversité des styles, eux-mêmes soumis à variations au cours de l'histoire, qui ont fleuri du nord-ouest au sud-est de cet archipel étalé sur plus de mille milles. Comme peut aussi le rappeler ce livre, le public occidental doit une très large part de sa connaissance des arts océaniens à des publications d'initiative privée, généreuses pour certaines, clairement intéressées pour beaucoup d'autres, tandis que les institutions publiques étaient et restent insuffisamment pourvues pour publier dignement les objets qu'elles conservent, même à l'heure des catalogues en ligne, non moins coûteux en temps de travail et d'étude 
que ceux de l'ère Gutenberg. Il serait absurde de se priver par attitude hypercritique de la documentation composite ainsi procurée, quoiqu'elle exige certainement un surcroît de vigilance si l'on souhaite en tirer parti.

Le terme de collection dont le titre de ce volume se prévaut est également à apprécier de façon nuancée. À titre commercial, Kevin Conru a fait paraître dans le dernier numéro de la revue du musée Barbier-Mueller une photographie de détail d'un objet des îles Salomon non décrit dans ce volume édité en 2008, et peut-être acquis ultérieurement (Conru, 2010 : 4). Cette pièce exceptionnelle, apparemment de première importance pour la connaissance des arts du centre de l'archipel où l'on peine à reconstituer la tradition des masques (au-delà par exemple du spécimen collecté en 1929 à Savo par Eugen Paravicini pour le musée de Bâle, Vb 6803), rejoindra-t-elle la collection Conru, ou telle autre collection continuant aujourd'hui à se nourrir sur le marché de l'art pour être publiée et vendue ensuite, à l'instar de ce qui s'est récemment présenté comme la meilleure collection privée au monde d'objets de Nouvelle-Guinée ? Pourtant ces collections en valent souvent bien d'autres ainsi dénommées dans les musées. C'est au hasard des escales de l'HMs CurAÇOA que fut rassemblée la « Julius L. Brenchley Collection » du British Museum si utilement décrite en 1987 par Deborah Waite pour ce qui concerne les arts des Salomon, et c'est la plupart du temps selon les occasions du marché de l'art que se constituent ou s'augmentent la plupart des collections d'aujourd'hui, publiques comme privées.

Comparé aux deux ouvrages précédents de D. Waite $(1983,1987)$ et aux rares publications ayant pour sujet l'ensemble des arts des îles Salomon (dont un bon catalogue d'un marchand d'art parisien [Carlier, 2001], oublié dans la bibliographie, p. 195sq.), ce volume présente un progrès manifeste, par le nombre et la qualité des illustrations dues à Hughes Dubois, l'un des meilleurs spécialistes actuels de la photographie d'objets, et par l'ampleur du texte continu qui les accompagne, tandis que les légendes ne livrent que des indications minimales. Du fait de ce probable parti pris de l'éditeur, D. Waite n'a pu consacrer aux pièces de cette collection le type de notice détaillée qui faisait le prix de sa première publication (1983), au moins aux yeux des spécialistes. Des descriptions approfondies n'auraient pas forcément découragé le grand public, elles auraient même pu l'éclairer sur nombre d'objets réunis dans ce volume, dont quelques-uns semblent inédits et dont d'autres sont au contraire connus de longue date sur le marché de l'art. Par exemple, une sculpture anthropomorphe accompagnée d'une figuration de chien, probablement destinée à la poupe d'un canot de San Cristobal $\left(\mathrm{n}^{\circ} 25\right.$ p. 57 , le texte p. 62 parle de « proue ») avait été très correctement photographiée et décrite dans un catalogue de vente (Christie's Londres, $1^{\text {er }}$ décembre 1982, lot 54, "small sections missing ») et il est dommage, dans une publication de cette ambition, que les informations données voilà un quart de siècle et les restaurations apportées à l'objet dans l'intervalle n'aient pas été signalées, même en bas de page.
D'autres objets peu fréquents dans les collections publiques ou privées auraient eux aussi mérité des précisions, tel le $n^{\circ} 75$, bâton de danse Kwaio (ethnonyme que ne cite pas D. Waite p. 131) de Malaita dont Pierre Maranda a collecté des spécimens plus récents (dispersés à Paris dans la vente Sotheby's du 15 juin 2004, lots 85 et suivants ; voir aussi Kaeppler, Kaufmann et Newton, 1993 : 455, fig. 500). Les spécialistes seront également déroutés par la numérotation obscure attribuée aux objets, ne reflétant apparemment ni la chronologie de leur acquisition ni un essai de classement typologique ou thématique, comme s'y efforce au contraire le texte de D. Waite, dont c'est l'un des réels apports à la recherche. Tout en suivant un fil géographique désormais classique menant de Nissan au nord-ouest à Santa Cruz au sud-est (avec des excursions jusqu'à Rennel et Ontong Java), elle s'est attachée à distinguer trois catégories d'objets, les sculptures anthropomorphes et zoomorphes, les « assemblages, récipients et véhicules », et enfin « tous les objets qui représentent le statut social », boucliers, armes, outils, parures et moyens d'échanges.

Elle ne dissimule pas les difficultés d'un tel classement, à commencer par les risques de chevauchements et de redites. Elle marque également son embarras, ou plutôt les lacunes des connaissances actuelles, à propos de sculptures anthropomorphes que beaucoup de connaisseurs tiennent pour des créations coloniales. « L'authenticité d'une œuvre n'est pas nécessairement dépendante d'une utilisation rituelle réelle », écrit-elle prudemment à propos d'une figure masculine de Buka (p. 22 et $\mathrm{n}^{\circ} 9$ p. 24), que suivent deux figurations féminines de Roviana ( $\mathrm{n}^{\circ} 10$ et 11 , pp. 27-29) dont elle ne manque pas d'interroger le « réalisme ». S'il semble à première vue folklorique et tardif, en rejeter en bloc le témoignage ne serait qu'une facilité hypercritique, explique-t-elle en substance. Voilà où cet ouvrage, plutôt destiné à faire connaître ces arts au grand public, intéressera réellement les spécialistes, qui devraient également avoir plaisir à découvrir ou à retrouver des objets excellemment photographiés, et judicieusement choisis par un collectionneur et marchand d'art qui avait déjà manifesté son attachement pour les îles Salomon, en republiant avec soin les photos un peu oubliées de Bernatzik (Conru et Coleman, 2002) chez le même éditeur.

\section{RÉFÉRENCES CITÉES}

CARLIER Jean-Édouard, 2001. Regard sur les îles Salomon, Paris, Galerie Voyageurs et Curieux.

Conru Kevin et Alan D. Coleman, 2002. Bernatzik: " Mers $d u$ Sud», Milan, 5 Continents.

Conru Kevin, 2010. New Georgia, Roviana Lagoon Wood Mask, Rokoroko, Arts et Cultures 11, p. 4.

KaEPPler Adrienne L, Christian KaufmanN et Douglas Newton, 1993. L'Art océanien, Paris, Citadelles \& Mazenod. 
Waite Deborah B., 1983. Art des îles Salomon dans les collections du musée Barbier-Mueller, Genève, musée Barbier-Mueller.

-, 1987. Artefacts from the Solomon Islands in the Julius Brenchley Collection, Londres, British Museum Publications.

Gilles Bounoure,

Paris

RaABE Eva Ch. [Herausgegeben von], 2008. Reisen und Entdecken. Vom Sepik an den Main. Hintergründe einer Ausstellung, Frankfurt am Main, Museum der Weltkulturen, 78 pages, bibliogr.., cartes, nombreuses photos couleurs et noir et blanc.

L'exposition qui s'est tenue du 27 octobre $2007 \mathrm{au}$ 30 août 2009 au Museum der Weltkulturen à Francfort avait pour ambition de présenter deux expéditions de collectes d'objets ethnographiques qui furent parmi les dernières à être organisées par un musée allemand dans la région du fleuve Sépik en Papouasie NouvelleGuinée. Cette exposition pose le problème de la collecte des artefacts à grande échelle dans les pays du Sud. Les expéditions de Francfort eurent lieu, en effet, plus de dix ans avant l'accession à l'indépendance de la Papouasie Nouvelle-Guinée. Depuis la fin du XIX ${ }^{\text {e }}$ siècle et jusqu'au traité de Versailles de 1919, la partie nord de ce pays fut une colonie de l'Empire germanique connue sous le nom de Kaiser WilhelmsLand. De nombreuses expéditions scientifiques y furent organisées, elles rapportèrent des milliers d'objets qui vinrent remplir les réserves des musées de folklore et d'ethnographie de nombreuses villes allemandes.

Le fleuve Sépik demeure une région mythique pour de nombreux collectionneurs d'objets océaniens par la profusion des styles et l'énorme production des artistes locaux en ce qui concerne architecture, sculpture, poterie, gravure, peinture et artisanats divers. Pourtant, il n'y a guère plus d'un siècle que les Européens découvrirent cette région. L'existence de ce fleuve fut signalée par divers navigateurs qui, depuis le $\mathrm{XVII}^{\mathrm{e}}$ siècle, longèrent la côte Nord de la grande île de Nouvelle-Guinée. Schouten et Lemaire évoquèrent dans leurs journaux l'embouchure d'un grand fleuve. Le Français Dumont d'Urville fit la même constatation au mois d'août 1827 en observant des changements de la coloration de l'eau de la mer. Mais aucun d'entre eux n'osa s'aventurer vers l'intérieur. Ce fut le Prussien Otto Finsch qui, le premier, en 1885, fit une reconnaissance sur vingt milles dans le delta de ce fleuve qu'il dénomma Kaiserin Augusta Fluss, du nom de l'épouse du Kaiser Wilhelm (l'empereur Guillaume). De nombreuses expéditions suivirent, organisées par les Allemands, les Américains, les Suisses, les Néerlandais, les Britanniques et même les Français. En effet, cinq jeunes Français avec un équipage de neuf marins naviguèrent en 1935 durant près d'un mois sur le fleuve à bord de leur yacht La Korrigane. Ils y collectèrent dans les villages plus de six cents objets divers dont un tiers se trouve actuellement conservé au musée du quai Branly (Coiffier, 2001). Ce sont donc plusieurs dizaines de milliers d'objets divers dont de nombreuses œuvres majeures qui furent exportés de cette région de Nouvelle-Guinée durant soixante années avant que la législation du nouvel État papou ne vienne essayer de mettre un frein à cette hémorragie dramatique pour les cultures locales. Le fleuve de l'impératrice Augusta, appelé Ambusat par les populations de la partie centrale, conserva son nom allemand jusqu'au début de la Première Guerre mondiale lorsque l'administration australienne prit dès 1914 la responsabilité d'administrer ce territoire. Elle préféra utiliser le terme local de Sipik ou Sépik qui est un des noms vernaculaires usuels pour désigner le fleuve dans sa partie inférieure.

Ce sont les deux expéditions du Suisse Alfred Bühler qui motivèrent celles du musée de Francfort. En effet, quelques années avant celles-ci, Alfred Bühler, directeur du Museum für Völkerkunde et professeur d'ethnologie à l'université de Bâle, organisa en 1955-1956 une grande expédition sur le Sépik en compagnie du photographe René Gardi. Lors de sa seconde expédition, plus ciblée sur l'étude des styles artistiques locaux, il était accompagné cette fois de l'historien d'art Anthony Forge. Le D ${ }^{\mathrm{r}}$ Adolf Ellegard Jensen, titulaire de la chaire d'ethnologie à l'université de Francfort, assurait à cette époque la direction conjointe de l'Institut Frobenius et du Museum für Völkerkunde de la ville de Francfort car ce musée avait perdu ses locaux d'exposition du fait des destructions de la guerre. Le $\mathrm{D}^{\mathrm{r}}$ Jensen se montra très impressionné par les résultats obtenus par Bühler et proposa à la municipalité de Francfort de cofinancer une expédition en Nouvelle-Guinée. Il obtint un budget conséquent de cent mille marks et demanda à deux assistants de l'Institut Frobenius, Eike Haberland et Meinhard Schuster, de l'organiser. L'expédition fut divisée en deux parties: la première, conduite par Meinhard Schuster, en 1961, se concentra sur tous les villages depuis les monts Washkuk en amont jusqu'au village d'Angoram en aval ainsi que sur la région du Korewori au sud, la seconde, en1963, dirigée par Eike Haberland, assisté de Siegfried Seyfarth, concentra ses efforts sur le haut Korewori. Schuster et Haberland rapportèrent de leurs séjours, qui durèrent plusieurs mois, plus de cinq mille objets dont certains de dimensions imposantes comme des tambours à fente et des piliers sculptés de maisons cérémonielles. Plusieurs d'entre eux furent exposés à l'extérieur du musée où ils furent endommagés par les intempéries et les gaz des automobiles. C'est donc une réplique d'un de ces poteaux, nommé Meriameï, qui fut dressée à l'occasion de l'exposition devant la façade du Museum der Weltkulturen comme élément d'appel pour les visiteurs. Avec trois autres piliers, il provient de la maison Mundjimbit du village de Kanganaman (pp. 26-27). Cet édifice cérémoniel ne fut jamais reconstruit par les villageois après le passage de l'expédition. Fort heureusement, sa voisine, la maison Wolimbi, se dresse toujours majestueusement au centre d'une large place d'herbe rase à une centaine de mètres du seul pilier de la maison Mundjimbit resté en 